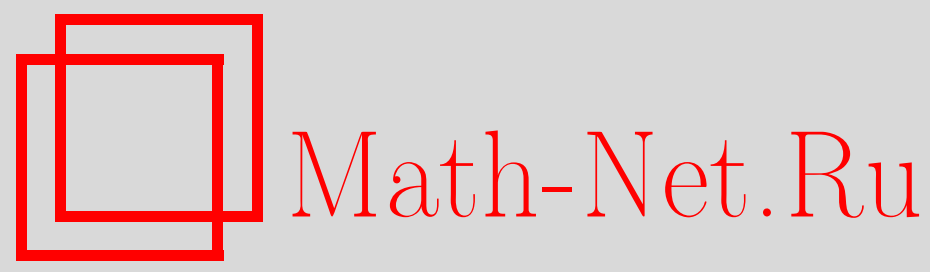

В. Н. Безверхний, И. В. Добрынина, Решение проблемы степенной сопряженности слов в группах Кокстера экстрабольшого типа, Дискрет. матем., 2008, том 20, выпуск 3, 101110

DOI: https://doi.org/10.4213/dm1017

Использование Общероссийского математического портала Math-Net.Ru подразумевает, что вы прочитали и согласны с пользовательским соглашением http://www . mathnet.ru/rus/agreement

Параметры загрузки:

IP : 54.80 .97 .219

26 апреля 2023 г., 15:35:39 


\title{
Решение проблемы степенной сопряженности слов в группах Кокстера экстрабольшого типа
}

\author{
() 2008 г. В. Н. Безверхний, И. В. Добрынина
}

\begin{abstract}
Для групп Кокстера экстрабольшого типа решены проблема степенной сопряженности слов и проблема пересечения циклических подгрупп, то есть предложен алгоритм, который по двум элементам находит порождающий элемент пересечения их циклических подгрупп.
\end{abstract}

Группа $G$, заданная системой образующих $a_{i}, i \in J,|J|<\infty$, и системой определяющих соотношений $a_{i}^{2}=1$ для всех $i \in J,\left(a_{i} a_{j}\right)^{m_{i j}}=1, i \neq j, i, j \in J, m_{i j}$ - элемент матрицы Кокстера $\left(m_{i j}\right), i, j \in J$, соответствующей данной группе [1], причем $m_{i j} \geqslant 3$ для $i \neq j$, называется группой Кокстера большого типа. В случае $m_{i j}>3$ эту группу называют группой Кокстера экстрабольшого типа.

Проблемы равенства и сопряженности слов в группах Кокстера экстрабольшого и большого типов решены в [1] и [2].

Целью работы является решение проблемы степенной сопряженности слов в группах Кокстера экстрабольшого типа. Кроме этого, для данного класса групп будет решена проблема пересечения циклических подгрупп.

Пусть $F_{i}=\left\langle a_{i} ; a_{i}^{2}\right\rangle, F=\prod_{i=1}^{n} * F_{i}-$ свободное произведение циклических групп порядка 2. Отождествим каждый образующий $a_{i}$ группы $F$ с его обратным $a_{i}^{-1}$. Слово $w=a_{i_{1}} \ldots a_{i_{n}}$ группы $F$ называется приведенным, если индексы рядом стоящих букв $a_{i_{j}}$ и $a_{i_{j+1}}$ записи $w$ различны; длина $w$ равна $n$. Пусть $m_{i j}<\infty$ и $r_{i j}=\left(a_{i} a_{j}\right)^{m_{i j}}$, тогда в $F$ существуют в точности две различные перестановки $r_{i j}: r_{i j}=\left(a_{i} a_{j}\right)^{m_{i j}}$ и $r_{j i}=\left(a_{j} a_{i}\right)^{m_{i j}} i \neq j$. Обозначим через $F_{i j}$ группу $F_{i j}=F_{i} * F_{j}$, через $G_{i j}$ группу Кокстера экстрабольшого типа $G_{i j}=\left\langle a_{i}, a_{j} ; a_{i}^{2}, a_{j}^{2}, r_{i j}, r_{j i}\right\rangle$.

Обозначим через $R_{i j}$ множество всех нетривиальных слов, циклически приведенных в свободном произведении $F_{i j}$ и равных 1 в группе $G_{i j}, R_{i j}=\left\{r_{i j}^{l}, r_{j i}^{m}\right\}, l, m \in \mathbf{N}$ (см. [2]). Элемент $r \in R_{i j}$ будем называть соотношением типа $(i, j)$.

В дальнейшем под $R$ будем понимать симметризованное подмножество

$$
R=\bigcup_{i, j \in J} R_{i j}
$$

свободного произведения $F$. Тогда группа Кокстера может быть задана представлением $G=\left\langle a_{i} ; a_{i}^{2}, R, i=1, \ldots, n\right\rangle$. Пусть $w$ - нетривиальное циклически приведенное в $F$ слово, равное единице в группе $G$ Кокстера экстрабольшого типа, то есть $w \in\langle R\rangle^{F}$, где $\langle R\rangle^{F}$ - нормальное замыкание симметризованного множества $R$ в свободном произведении $F$. Учитывая, что $a_{i}=a_{i}^{-1}$ в $F$ можно строить $R$-диаграммы над $F$ в точности так 
же, как над свободной группой [3], областями этих диаграмм являются $R_{i j}$-диаграммы с метками типа $(i, j)$.

Подвергнем $R$-диаграмму $M$ следующему преобразованию. Если две области $D_{1}, D_{2}$ являются одновременно $R_{i j}$-диаграммами и пересекаются по ребру с меткой $\varphi\left(\partial D_{1} \cap \partial D_{2}\right)$, то, стирая это ребро, объединим $D_{1}, D_{2}$ в одну область $D$. При этом возможно, что метка границы полученной области равна единице в свободном произведении $F$. Тогда, удалив эту область, склеиваем ее границу. Таким образом, через конечное число шагов мы получим приведенную (в $F$ ) связную односвязную $R$-диаграмму $M$, инвариантную относительно рассмотренного преобразования с граничной меткой, равной $w$, причем если две области $D^{\prime}, D^{\prime \prime}$ из $M$ пересекаются по ребру, то длина метки этого ребра равна единице. Каждая приведенная связная односвязная $R$-диаграмма $M$ группы Кокстера экстрабольшого типа удовлетворяет условию $C(8)$.

Обозначим через $\partial M$ граничный цикл $M$. Область $D \subset M$ назовем граничной, если $\partial M \cap \partial D \neq \varnothing$. Символом $i(D)$ будем обозначать число внутренних ребер в граничном цикле $D$, символом $d(D)$ - число всех ребер в граничном цикле $D$, символом $d(v)-$ степень вершины $v$, а $|w|$ - длину слова $w$.

Будем говорить, что $\partial D \cap \partial M$ есть правильная часть $M$, если $\partial D \cap \partial M$ есть объединение последовательности $l_{1}, \ldots, l_{n}$ замкнутых ребер, где $l_{1}, \ldots, l_{n}$ встречаются в данном порядке в некотором граничном цикле для $D$ и в некотором граничном цикле для $M$.

Граничную область $D \quad R$-диаграммы $M$ назовем простой, если $\partial D \cap \partial M$ есть правильная часть. Простая область $D$ диаграммы $M$ называется деновской, если $i(D)<d(D) / 2$.

Связная односвязная диаграмма $M$ называется диском, если ее граничный цикл $\partial M$ - простая замкнутая кривая.

Определение 1. Пусть $M_{1}$ - приведенная связная, односвязная поддиаграмма $R$-диаграммы $M$ группы Кокстера экстрабольшого типа с границей $\partial M_{1}=e_{1} \gamma e_{2} \delta$, где $e_{1}-$ ребро $A B, \gamma-$ путь $B C, e_{2}-$ ребро $C D, \delta-$ путь $D A$. Тогда последовательность областей $D_{1}, D_{2}, \ldots, D_{n}$ из $M_{1}, e_{1} \in D_{1}, e_{2} \in D_{n}, n \geqslant 2$, образует полосу в $M$, если

(1) $\partial D_{i} \cap \gamma, \partial D_{i} \cap \delta-$ правильная часть $M_{1}$ для всех $i, 1 \leqslant i \leqslant n$;

(2) для всех $i, 1 \leqslant i<n$, границы областей $D_{i}$ и $D_{i+1}$ пересекаются по ребру;

(3) $\left|\partial D_{1} \cap \gamma\right|=\left|\partial D_{1} \cap \delta\right|+2,\left|\partial D_{n} \cap \gamma\right|=\left|\partial D_{n} \cap \delta\right|+2$ и $\left|\partial D_{j} \cap \gamma\right|=\left|\partial D_{j} \cap \delta\right|$, $2 \leqslant j<n$.

Удаление деновской области диаграммы $M$, то есть удаление ее граничного пути, называется деновским сокращением диаграммы $M$ или $R$-сокращением. Будем говорить, что $M$ является $R$-приведенной, если она не содержит деновских областей.

Пусть $\Pi-$ полоса диаграммы $M$. Замену диаграммы $M$ на диаграмму $M_{1}$, полученную из $M$ удалением полосы П, назовем специальным $R$-сокращением или $\bar{R}$-сокращением. Если $M$ не содержит полос, то назовем $M$ специально $R$-приведенной или $\bar{R}$-приведенной. Слово $w \in G$ назовем $R$-приведенным, если $w$ является граничной меткой приведенной (в $F$ ) диаграммы, не содержащей деновских областей. Назовем $w$ циклически $R$-приведенным, если все его циклические перестановки являются $R$-приведенными словами.

Циклически $R$-приведенное слово $w$ группы $G$ Кокстера экстрабольшого типа назовем $\bar{R}$-приведенным, если $w$ является граничной меткой приведенной диаграммы, не содержащей полос. Если $w^{\prime}$ получено из $w R$ - или $\bar{R}$-приведением, то $\left|w^{\prime}\right|<|w|$. 
Теорема 1 ([2]). Сущчествует алгоритм, позволяющий для любого цииклически приведенного слова w группь Кокстера экстрабольшого типа выяснить, является ли $w R$ - или $\bar{R}$-приведенным.

Рассмотрим теперь кольцевые диаграммы. Назовем связную карту $M$ на плоскости $E^{2}$ кольцевой картой, если дополнение ее состоит в точности из двух компонент. Через $K$ обозначим неограниченную, а через $H$ - ограниченную компоненты множества $M$. Назовем $\partial M \cap \partial K$ внешней, а $\partial M \cap \partial H-$ внутренней границей карты $M$. Цикл минимальной длины, содержащий все ребра внешней границы карты $M$, есть внешний граничный цикл карты $M$, обозначим его $\sigma$. Аналогично определяется внутренний граничный цикл карты $M$. Обозначим его $\tau$.

Пусть $F$ - свободное произведение, $R$ - симметризованное подмножество его элементов, $N=\langle R\rangle^{F}$ - нормальное замыкание $R$ в $F$. Пусть $u$ и $z-$ два циклически приведенных слова из $F$, не лежащих в $N$ и не сопряженных в $F$. Если $u$ и $z$ представляют собой некоторые сопряженные элементы группы $G=F / N$, то существует приведенная кольцевая $R$-диаграмма $M$, содержащая не менее одной области, такая, что если $\sigma$ и $\tau-$ соответственно внутренний и внешний граничные циклы карты $M$, причем $\varphi(\sigma)=u$, а $\varphi(\tau)=z^{-1}$ (см. [3]). Пусть $u, z$ - два циклически приведенных слова, $u, z \notin\langle R\rangle^{G}$, и не сопряжены в $F$ и сопряжены в $G$. Тогда существует связная приведенная кольцевая $R$-диаграмма $M$ с внешней граничной меткой $u$ и внутренней граничной меткой $z^{-1}$, граничными метками областей $D$ которой являются соотношения из $R$. Подвергнем кольцевую $R$-диаграмму следующему преобразованию. Если две области $D_{1}, D_{2}$ пересекаются по ребру $e$, причем общей граничной меткой является либо соотношение из некоторого симметризованного множества $R_{i j}$, либо слово, равное 1 в $F_{i j}$, то стерев ребро $e$, объединяем $D_{1}$ и $D_{2}$ в одну область, причем в случае равенства единице в $F_{i j}$, данную область вырезаем, а ее границу склеиваем. В результате получаем кольцевую $R$-диаграмму $M^{\prime}$ с теми же граничными метками $u$ и $z^{-1}$. В дальнейшем будем рассматривать кольцевые связные приведенные $R$-диаграммы $M$, инвариантные относительно указанных выше преобразований. Будем предполагать также, что $u$ и $z^{-1}$ являются циклически $R$-приведенными и $\bar{R}$-приведенными словами. Заметим, что меткой каждого ребра будет буква $a_{j}$.

Кольцевую связную приведенную $R$-диаграмму $M$ сопряженности слов $\varphi(\sigma), \varphi(\tau) \in G$, где $\sigma, \tau$ - соответственно внешний и внутренний граничный циклы $M$, назовем минимальной, если не существует кольцевой $R$-диаграммы $M_{0}$ с теми же граничными метками $\varphi(\sigma), \varphi(\tau)$, имеющей меньшее число областей.

Кольцевую связную приведенную однослойную $R$-диаграмму $M$ с граничными циклами $\sigma, \tau$ группы Кокстера большого типа, метки которой $\varphi(\sigma), \varphi(\tau)$ приведены в $F$, циклически $R$ - и $\bar{R}$-приведены, назовем особо специальной $R$-диаграммой, если в $M$ существует одна область $D$ такая, что

$$
|\varphi(\partial D \backslash(\partial D \cap \sigma))|=|\varphi(\partial D \cap \sigma)|,
$$

а для остальных областей $D^{\prime}$

$$
\left|\varphi\left(\partial D^{\prime} \backslash\left(\partial D^{\prime} \cap \sigma\right)\right)\right|=\left|\varphi\left(\partial D^{\prime} \cap \sigma\right)\right|+2 .
$$

Замену слова $\varphi(\sigma)$ словом $\varphi(\tau)$ назовем специальным кольцевым $R$-сокращением.

Определение 2. Будем говорить, что циклически несократимое слово $w$ группы Кокстера экстрабольшого типа обладает свойством $s$, если $w$ циклически $R$ - или $\bar{R}$-несократимо и к нему неприменимо кольцевое специальное $R$-сокращение. 
Согласно [2], существует алгоритм, позволяющий для любого циклически $R$ - или $\bar{R}$-приведенного слова $w$ из группы Кокстера большого типа установить, применимо ли к нему специальное кольцевое $R$-сокращение.

Область $D$ с граничным циклом $\partial D=e \gamma e^{-1} \delta$, расположенная по обе стороны относительно ребра $e$, в которой склеенные ребра $e$ и $e^{-1}$ пересекают граничный цикл $D$, называется $(s-i)$-областью.

Лемма 1 ([2]). Если $M$ - приведенная связная односвязная R-диаграмма над группой Кокстера экстрабольшого типа, то она не содержит $(s-i)$-областей.

Лемма 2. Пусть $M-$ приведенная связная односвязная $R$-диаграмма над группой Кокстера экстрабольшого типа, $\sigma$ - граничный ичикл $M$, слово $\varphi(\sigma)$ является $R$ - $u \bar{R}$-несократимым. Тогда М является однослойной.

Доказательство. По лемме 1 диаграмма $M$ не содержит $(s-i)$-областей. Допустим, что $M$ не является однослойной. Пусть $M$ содержит внутреннюю область $D^{\prime}$, причем $i\left(D^{\prime}\right) \geqslant 8$.

Группы Кокстера экстрабольшого типа являются частным случаем групп Кокстера большого типа, причем каждая приведенная связная односвязная $R$-диаграмма $M$ группы Кокстера большого типа удовлетворяет условию $C(6)$ (см. [1]).

Известно [3], что $[p, q]$-картой $((p, q)$-картой) называется карта, все внутренние вершины которой имеют степень не менее $p$, а все (внутренние) области имеют степень не менее $q$. Карты $C(6)$ есть карты с условием $(3,6)$. Пусть $M$ - кольцевая карта с условием $(3,6)$, тогда $M^{*}$, дуальная к ней карта, будет картой с условием $[6,3]$, для которой справедливо [3] соотношение

$$
6\left(Q_{M^{*}}-h_{M^{*}}\right) \leqslant \sum_{M^{*}}^{\bullet}(4-d(v))+\sum_{M^{*}}^{\circ}(6-d(v))+2 \sum_{M^{*}}(3-d(D))+2\left(V_{M^{*}}^{\bullet}-E_{M^{*}}^{\bullet}\right),
$$

где в $\sum_{M^{*}}^{\bullet}(4-d(v))$ суммирование проводится по граничным вершинам $v$ карты $M^{*}$, в $\sum_{M^{*}}^{\circ}(4-d(v))-$ по внутренним вершинам $v$ карты $M^{*}$, в $\sum_{M^{*}}(3-d(D))-$ по областям $D$ карты $M^{*}, V_{M^{*}}^{\bullet}-$ число граничных вершин карты $M^{*}, E_{M^{*}}^{\bullet}-$ число граничных ребер карты $M^{*}, Q_{M^{*}}$ - число компонент карты $M^{*}, h_{M^{*}}$ - число дырок карты $M^{*}$. Так как $M^{*}$ имеет не более одной дырки, $6\left(Q_{M^{*}}-h_{M *}\right) \geqslant 0$. Каждая область карты $M$ имеет внутренние ребра, $M^{*}$ не имеет изолированных вершин, поэтому $V_{M^{*}}^{\bullet}-E_{M^{*}}^{\bullet} \leqslant 0$. Таким образом,

$$
\sum_{M^{*}}^{\bullet}(4-d(v))+\sum_{M^{*}}^{\circ}(6-d(v))+2 \sum_{M^{*}}(3-d(D)) \geqslant 0 .
$$

Рассматривая $M^{*}$ без граничных вершин степеней 1 и 2, получаем, что

$$
\sum_{M^{*}}^{\circ}(4-d(v)) \leqslant 0, \quad \sum_{M^{*}}^{\circ}(6-d(v))<0, \quad \sum_{M^{*}}(3-d(D)) \leqslant 0,
$$

что невозможно. Используя соответствие между картой и дуальной картой, для карты $M$ получаем противоречивость формулы

$$
\sum_{M}^{\bullet}(4-i(D))+\sum_{M}^{\circ}(6-d(D))+2 \sum_{M}(3-d(v)) \geqslant 0 .
$$

Таким образом, $M$ не содержит внутренних областей. 
Покажем, что $M$ не является двуслойной. Как замечено ранее, смежные области пересекаются по ребру, меткой которого является буква. Граница каждой области имеет меткой слово на двух образующих. Рассмотрим последовательные области $D_{1}, D_{2}$ и $D_{3}$, $D_{4}, D_{5}$, причем $D_{1}, D_{2}$ расположены в одном слое, а $D_{3}, D_{4}, D_{5}$ в другом и

$$
\partial D_{1} \cap \partial D_{2}=e_{1}, \quad \partial D_{1} \cap \partial D_{4}=e_{2}, \quad \partial D_{2} \cap \partial D_{4}=e_{3} .
$$

Области $D_{1}, D_{2}$ не образуют полосу, поэтому либо область $D_{3}$, соседняя с $D_{4}$, пересекается минимум по двум ребрам с $D_{1}$, либо область $D_{5}$, соседняя с $D_{4}$, пересекается минимум по двум ребрам с $D_{2}$, что невозможно. Лемма доказана.

Аналогично получаем следующее утверждение.

Лемма 3. Пусть $M$ - приведенная связная кольцевая диаграмма сопряженности слов $\varphi(\sigma), \varphi(\tau) \in G$ над группой Кокстера экстрабольшого типа, не содержашая $(s-i)$-областей, $\sigma$, $\tau$ - соответственно внешний и внутренний граничный ичикль $M$, слова $\varphi(\sigma)$, $\varphi(\tau)$ изиклически $R$ - и $\bar{R}$-несократимы. Тогда $M$ является однослойной.

Лемма 4 ([2]). Пусть $M$ - кольцевая связная приведенная минимальная $R$-диаграмма группь $G$ Кокстера экстрабольшого типа с граничными ичиклами $\sigma u \tau ; \varphi(\sigma), \varphi(\tau)$ удовлетворяют условию $s$. Тогда если $M$ содержит $(s-i)$-область, то все области $M$ являются $(s-i)$-областями и $\varphi(\sigma)=x, \varphi(\tau)=y, x, y \in\left\{a_{1}, \ldots, a_{n}\right\}$, где $\left\{a_{i}\right\}$, $i=1, \ldots, n,-$ множества образующих группьь $G$.

Лемма 5 ([4]). Пусть $G$ - группа Кокстера экстрабольшого типа с множеством образуюших $A$. Если $v_{0} \in G, v_{0} \neq 1$ в $G$ и $v_{0}-R$-, $\bar{R}$-приведенное слово, равное слову из подгруппь $G_{j}$ с образующими $A_{j}, A_{j} \subset A$, то $v_{0}-$ слово на образующих $A_{j}$.

Лемма 6 ([4]). Пусть $u, v \in G, G$ - группа Кокстера экстрабольшого типа, $A-$ множество образуюших $G, u$ u $v$ обладают свойством $s$ и сущчествует $z \in G$ такое, что $z^{-1} u z=v$. Тогда, если слово и есть слово на образующих $A_{j}, A_{j} \subset A, u$ не является образующим из $A_{j}$, то $v \in G_{j}$ и существует $z^{\prime} \in G_{j}, z^{\prime}=z$ в $G$ такое, что $z^{\prime}-1 u z^{\prime}=v$.

Лемма 7 ([5]). Слово $w$ группь Кокстера экстрабольшого типа G имеет конечный порядок тогда и только тогда, когда оно сопряжено с некоторым словом $w^{\prime} \in G_{a b}=\left\langle a, b ;(a b)^{m_{a b}}=1, a^{2}=b^{2}=1\right\rangle$.

Лемма 8 ([6]). Пусть слово $w \in G$ обладает свойством s и имеет бесконечный порядок. Тогда существует слово, сопряженное $w$ или $w^{2}$ в группе $G$, любая степень которого ичиклически $R$ - и $\bar{R}$-несократима.

Определение 3. Будем говорить, что в группе $G$ разрешима проблема слабой степенной сопряженности слов, если существует алгоритм, позволяющий для любых слов $w, v \in G$ таких, что $w \notin\langle v\rangle$, установить, существует ли целое число $n$ такое, что слова $w^{n}, v$ сопряжены в группе $G$.

Теорема 2 ([7]). В группе Кокстера экстрабольшого типа разрешима проблема слабой степенной сопряженности слов.

Определение 4. Будем говорить, что в группе $G$ разрешима проблема степенной сопряженности слов, если существует алгоритм, позволяющий для любых слов $w, v \in G$ установить, существуют ли ненулевые целые числа $n, m$ такие, что слова $w^{n}, v^{m}$ сопряжены в группе $G$. 
Теорема 3. В группе Кокстера экстрабольшого типа разрешима проблема степенной сопряженности слов.

Доказательство. Если $w=x, x \in\left\{a_{i}\right\}_{i=1}^{n}$, то из леммы 4 следует, что $v=y, y \in\left\{a_{i}\right\}_{i=1}^{n}$, диаграмма сопряженности этих слов состоит из $(s-i)$-областей, и доказательство теоремы сводится к решению проблемы сопряженности [2].

Если одно из слов $w, v$ имеет конечный порядок, то решение проблемы степенной сопряженности для этих слов следует из лемм $6,7$.

Пусть слова $w, v$ имеют бесконечный порядок. Если необходимо, перейдем от слов $w, v$ к сопряженным с ними или их квадратами словам $w_{0}, v_{0}$, любая степень которых циклически $R$ - и $\bar{R}$-несократима (на основании леммы 8). Рассмотрим приведенную диаграмму $M$ сопряженности слов $w_{0}^{n}, v_{0}^{m}$ с границей $\partial M=\sigma \cup \tau$, причем $\varphi(\sigma)=w_{0}^{n}$, $\varphi(\tau)=v_{0}^{-m}$. Будем считать, что $m, n-$ минимальные числа, то есть из $M$ нельзя вырезать поддиаграмму, замкнув которую в кольцо, получим диаграмму степенной сопряженности слов $w_{0}, v_{0}$. Покажем, что числа $m, n$ можно ограничить. Для доказательства достаточно рассмотреть случай, когда $\left|w_{0}\right|=\left|v_{0}\right|$. Общий случай сводится к данному при рассмотрении соответствующих минимальных степеней $w_{0}, v_{0}$, для которых выполняется условие равенства длин.

Рассмотрим случай кольцевой диаграммы. Она является однослойной по лемме 3. Заметим, что любая область $D$ содержит ребра как на $\sigma$, так и на $\tau$, причем между любыми двумя областями, имеющими на $\sigma(\tau)$ на два ребра меньше, чем на $\tau(\sigma)$, содержится область, имеющая на $\sigma(\tau)$ на два ребра больше, чем на $\tau(\sigma)$, так как в противном случае в $M$ содержится полоса. Кроме того, в диаграмме не может быть только одна область, имеющая на $\sigma(\tau)$ на два ребра больше, чем на $\tau(\sigma)$, ибо, склеив два экземпляра диаграммы $M$, получим $\bar{R}$-сократимость квадрата граничной метки $M$, что невозможно.

Допустим, что существует область $D_{1}$ такая, что

$$
\left|\varphi\left(\partial D_{1} \cap \sigma\right)\right|=\left|\varphi\left(\partial D_{1} \cap \tau\right)\right|+2 .
$$

Пусть $w_{0}^{*}$ - циклическая перестановка слова $w_{0}$, причем $\varphi\left(\partial D_{1} \cap \sigma\right)$ - начало слова $w_{0}^{*}$. Рассмотрим поддиаграмму $L=D_{1} \cup D_{2} \cup \ldots \cup D_{r}$ с минимальным $r$ такую, что $w_{0}^{*}$ является подсловом слова $u \equiv \varphi(\partial L \cap \sigma)$. Возможны следующие случаи.

1. $\left|w_{0}\right|=|u|$. Наклеим область $D_{1}$ по границе $\sigma$ на область $D_{r+1}$. Тогда слова $\varphi\left(D_{1}\right)$ и $\varphi\left(D_{r+1}\right)$ взаимно обратны. Следовательно,

$$
\begin{aligned}
\varphi\left(\partial D_{1} \cap \sigma\right) & \equiv \varphi\left(\partial D_{r+1} \cap \sigma\right), \\
\varphi\left(\partial D_{1}\right) & \equiv \varphi\left(\partial D_{r+1}\right) .
\end{aligned}
$$

Обозначим через $D_{0}$ область, предшествующую $D_{1}$. Справедливо равенство

$$
\varphi\left(\partial D_{0} \cap \partial D_{1}\right) \equiv \varphi\left(\partial D_{r} \cap \partial D_{r+1}\right) .
$$

Склеим ребра $\partial D_{0} \cap \partial D_{1}, \partial D_{r} \cap \partial D_{r+1}$ поддиаграммы $L$. Получим сопряженность $w_{0}^{*}, v_{0}^{*}$ и, следовательно, сопряженность $w_{0}, v_{0}$.

2. $\left|w_{0}\right|<|u|$. Наклеим область $D_{1}$ по границе $\sigma$ на пару областей $D_{r}, D_{r+1}$. В данном случае либо метка $\varphi\left(D_{0}\right)$ области $D_{0}$, предшествующей $D_{1}$, либо метка $\varphi\left(D_{r+1}\right)$ области $D_{r+1}$ сократимы в $F$, что невозможно. 
Если для любой области $D$ справедливо равенство

$$
|\varphi(\partial D \cap \sigma)|=|\varphi(\partial D \cap \tau)|,
$$

то в силу того, что метка каждого ребра есть буква и области пересекаются по одному ребру, получаем, что

$$
\varphi(\partial D \cap \sigma) \equiv \varphi(\partial D \cap \tau) .
$$

Таким образом, $w_{0} \equiv v_{0}$ и, следовательно, $w_{0} \sim v_{0}$.

Рассмотрим теперь простые кольцевые диаграммы сопряженности слов $w_{0}^{n}, v_{0}^{m}$.

Лемма 9. Пусть

$$
M=\left(\bigcup_{i=1}^{p} N_{i}\right) \cup\left(\bigcup_{j=1}^{p} \gamma_{j}\right)
$$

- простая кольцевая диаграмма сопряженности ичклически $R$-, $\bar{R}$-несократимых слов $w_{0}^{n}, v_{0}^{m}$, где $m, n-$ наименьшие числа с таким свойством, $N_{i}-$ поддиаграммы (диски) в М с границами $\partial N_{i}=\sigma_{i} \cup \tau_{i}, \sigma_{i} \cap \tau_{i}=\left\{A_{i}, B_{i}\right\}-$ вершины, $i=1, \ldots, p, \gamma_{i}-$ простые пути с концами $B_{i-1}, A_{i}, i=2, \ldots, p$, простой путь $\gamma_{1}$ имеет начало $B_{p}$ и конец $A_{1}$. Тогда $\left|\gamma_{i}\right|<\left|w_{0}\right|, p \leqslant\left|w_{0}\right|^{2}$.

Доказательство. Предположение, что $\left|\gamma_{i}\right| \geqslant\left|w_{0}\right|$ при $i=1, \ldots, p$ приводит к тому, что циклические перестановки слов $w_{0}, v_{0}$ совпадают и, следовательно, слова $w_{0}, v_{0}$ сопряжены, что противоречит минимальности чисел $m, n$.

Покажем, что $p \leqslant\left|w_{0}\right|^{2}$. Пронумеровывая буквы в словах $w_{0}, v_{0}$, получаем $w_{0}=x_{1} x_{2} \ldots x_{\left|w_{0}\right|}, v_{0}=y_{1} y_{2} \ldots y_{\left|v_{0}\right|}$. Среди этих букв могут встречаться одинаковые, но мы будем рассматривать вхождения этих букв, поэтому буквы, стоящие на разных местах будем считать разными.

Рассмотрим метки ребер $e_{i_{1}} \in \sigma_{i}$ и $e_{i_{2}} \in \tau_{i}$ диаграмм $N_{i}$, прилегающих к вершинам $B_{i}$. Метками $e_{i_{1}}$ являются буквы $x_{i_{1}}$. Существует не более $\left|w_{0}\right|$ различных букв $x_{i_{1}}$. Если $p>\left|w_{0}\right|$, то хотя бы одна из этих букв повторяется. Но повторяться каждая буква может не более, чем $\left|v_{0}\right|$ раз, так как иначе повторится соответствующая метка $x_{i_{2}}$ ребра $e_{i_{2}}$ и из $M$ можно вырезать поддиаграмму так, что, замкнув оставшуюся часть в кольцо, получим диаграмму, удовлетворяющую условию леммы, а это невозможно в силу минимальности чисел $m, n$. Итак, $p \leqslant\left|w_{0}\right|\left|v_{0}\right|$, и так как $\left|w_{0}\right|=\left|v_{0}\right|$, справедливо неравенство $p \leqslant\left|w_{0}\right|^{2}$.

Лемма доказана.

Будем говорить, что диск $N_{i}$ с границей $\partial N_{i}=\sigma_{i} \cup \tau_{i}, \sigma_{i} \cap \tau_{i}=\left\{A_{i}, B_{i}\right\}$, является длинным, если он имеет метку $\varphi\left(\sigma_{i}\right)$ такую, что $\left|\varphi\left(\sigma_{i}\right)\right| \geqslant 4\left|w_{0}\right|$, и коротким в противном случае. На основании леммы 9 в случае, когда все диски диаграммы $M$ короткие, числа $m, n$ можно ограничить. Рассмотрим длинный диск $N_{i}$. Допустим, что в $N_{i}$ существует область $D_{1}$ такая, что

$$
\left|\varphi\left(\partial D_{1} \cap \sigma_{i}\right)\right|=\left|\varphi\left(\partial D_{1} \cap \tau_{i}\right)\right|+2
$$

Начиная с данной области повторим рассуждения, проведенные для кольцевой диаграммы. Получим противоречие с минимальностью чисел $m, n$. Если в $N_{i}$ для любой области $D$, за исключением областей, содержащих вершины $A_{i}, B_{i}$, справедливо равенство

$$
|\varphi(\partial D \cap \sigma)|=|\varphi(\partial D \cap \tau)|,
$$


то из приведенных выше рассуждений также получим противоречие с минимальностью чисел $m, n$. Таким образом, случай, когда в диаграмме $M$ существуют длинные диски, невозможен. Теорема доказана.

Определение 5. Будем говорить, что в группе $G$ разрешима проблема пересечения циклических подгрупп, если существует алгоритм, позволяющий для любых слов $w, v \in G$ установить, пусто или нет пересечение циклических подгрупп, порожденных в $G$ данными словами.

Теорема 4. В группе Кокстера экстрабольшого типа разрешима проблема пересечения ичиклческих подгрупп. Сущеествует алгоритм, выписывающий образующие данного пересечения.

Доказательство. Рассмотрим пересечение циклических подгрупп $\langle w\rangle$ и $\langle v\rangle$. Если одно из слов $w, v$ имеет конечный порядок, то решение проблемы пересечения циклических подгрупп следует из лемм 4-7.

Пусть слова $w, v$ имеют бесконечный порядок и в группе Кокстера экстрабольшого типа $G$ существует такое нетривиальное $t$, что $t=w^{n}=v^{m}$ для некоторых $m, n$. Будем считать, что $m, n-$ минимальные числа с таким свойством. Покажем, что $m$, $n$ можно ограничить. Заменим $w, v$ на сопряженные с ними слова $w^{\prime}, v^{\prime}$, обладающие свойством $s$. Перейдем от слов $w^{\prime}, v^{\prime}$ к сопряженным с ними или их квадратами словам $w_{0}, v_{0}$, любая степень которых циклически $R$ - и $\bar{R}$-несократима (на основании леммы 8 ). Получим $w_{0}^{n}=z^{-1} v_{0}^{m} z$. Считаем $z$ минимальным в классе $\left\langle w_{0}\right\rangle z\left\langle v_{0}\right\rangle$. Для доказательства достаточно рассмотреть случай, когда $\left|w_{0}\right|=\left|v_{0}\right|$. Общий случай сводится к данному при рассмотрении соответствующих минимальных степеней $w_{0}, v_{0}$, для которых выполняется условие равенства длин.

Возможны два случая, $|z| \neq 0$ и $|z|=0$.

Рассмотрим первый случай. Пусть $M-$ приведенная диаграмма сопряженности слов $w_{0}^{n}, v_{0}^{m}$ словом $z$ с границей $\partial M=\sigma \cup \tau$, причем $\varphi(\sigma)=w_{0}^{n}, \varphi(\tau)=v_{0}^{-m}$. Доказательство данного случая аналогично доказательству теоремы 3 . Действительно, рассмотрим случай кольцевой диаграммы. Она является однослойной по лемме 3.

Допустим, что существует область $D_{1}$ такая, что

$$
\left|\varphi\left(\partial D_{1} \cap \sigma\right)\right|=\left|\varphi\left(\partial D_{1} \cap \tau\right)\right|+2 .
$$

Пусть $w_{0}^{*}$ - циклическая перестановка слова $w_{0}$, причем $\varphi\left(\partial D_{1} \cap \sigma\right)-$ начало слова $w_{0}^{*}$. Рассмотрим поддиаграмму $L=D_{1} \cup D_{2} \cup \ldots \cup D_{r}$ с минимальным $r$ такую, что $w_{0}^{*}$ является подсловом слова $u \equiv \varphi(\partial L \cap \sigma)$. Возможны следующие подслучаи.

1. $\left|w_{0}\right|=|u|$. Наклеим область $D_{1}$ по границе $\sigma$ на область $D_{r+1}$. Тогда слова $\varphi\left(D_{1}\right)$ и $\varphi\left(D_{r+1}\right)$ взаимно обратны. Следовательно,

$$
\begin{aligned}
\varphi\left(\partial D_{1} \cap \sigma\right) & \equiv \varphi\left(\partial D_{r+1} \cap \sigma\right), \\
\varphi\left(\partial D_{1}\right) & \equiv \varphi\left(\partial D_{r+1}\right) .
\end{aligned}
$$

Аналогично,

$$
\varphi\left(\partial D_{2}\right) \equiv \varphi\left(\partial D_{r+2}\right), \ldots, \varphi\left(\partial D_{r}\right) \equiv \varphi\left(\partial D_{2 r}\right),
$$

и так далее, то есть области поддиаграммы $L$ образуют период. Обозначим через $D_{0}$ область, предшествующую $D_{1}$. Справедливо соотношение

$$
\varphi\left(\partial D_{0} \cap \partial D_{1}\right) \equiv \varphi\left(\partial D_{r} \cap \partial D_{r+1}\right) .
$$


Вырежем поддиаграмму $L$ и в диаграмме $M_{1}=M \backslash L$ склеим ребра $\partial D_{0} \cap \partial D_{1}$ и $\partial D_{r} \cap \partial D_{r+1}$. Получим приведенную диаграмму степенной сопряженности слов $w_{0}, v_{0}$ словом $z$, то есть числа $m, n$ можно уменьшить, что невозможно в силу их минимальности. Поэтому в данном случае $w_{0}=z^{-1} v_{0} z$.

2. $\left|w_{0}\right|<|u|$. Наклеим область $D_{1}$ по границе $\sigma$ на пару областей $D_{r}, D_{r+1}$. Но тогда либо метка $\varphi\left(D_{0}\right)$ области $D_{0}$, предшествующей $D_{1}$, либо метка $\varphi\left(D_{r+1}\right)$ области $D_{r+1}$ сократимы в $F$, что невозможно.

Если для любой области $D$ справедливо равенство

$$
|\varphi(\partial D \cap \sigma)|=|\varphi(\partial D \cap \tau)|,
$$

то в силу того, что метка каждого ребра есть буква и области пересекаются по одному ребру, получаем, что

$$
\varphi(\partial D \cap \sigma) \equiv \varphi(\partial D \cap \tau)
$$

Таким образом, $w_{0} \equiv v_{0}$ и, следовательно, как и в п. 1 , числа $m, n$ можно уменьшить. Таким образом, $w_{0}=z^{-1} v_{0} z$.

Рассмотрим теперь простую кольцевую диаграмму сопряженности слов $w_{0}^{n}, v_{0}^{m}$ словом z. Число дисков и простых путей можно ограничить согласно лемме 9. В случае, когда все диски диаграммы $M$ короткие, числа $m, n$, очевидно, можно ограничить. Рассмотрим длинный диск $N_{i}$. Допустим, что в $N_{i}$ существует область $D_{1}$ такая, что

$$
\left|\varphi\left(\partial D_{1} \cap \sigma_{i}\right)\right|=\left|\varphi\left(\partial D_{1} \cap \tau_{i}\right)\right|+2 .
$$

Начиная с данной области, повторим рассуждения, проведенные для кольцевой диаграммы. В результате получим, что диск $N_{i}$ может быть только коротким. Если в $N_{i}$ для любой области $D$, за исключением областей, содержащих вершины $A_{i}, B_{i}$, справедливо равенство

$$
|\varphi(\partial D \cap \sigma)|=|\varphi(\partial D \cap \tau)|,
$$

то также получим, что диск $N_{i}$ может быть только коротким.

Рассмотрим второй случай. Рассмотрим приведенную связную односвязную $R$-диаграмму $M$ с граничным циклом $\gamma \cup \delta$ такую, что

$$
\varphi(\partial M)=\varphi(\gamma) \varphi(\delta)=w_{0}^{n} v_{0}^{-m},
$$

где $\gamma, \delta$ гомеоморфны отрезку.

Рассмотрим случай, когда $M$ является диском, тогда $\partial M=\gamma \cup \delta, \gamma \cap \delta=\{A, B\}$. По лемме 2 эта диаграмма однослойна. Для диаграммы $M$ повторим все рассуждения, проведенные для диаграммы $N_{i}$ из первого случая. Получим, что диск $N_{i}$ может быть только коротким.

Случай, когда $M$ представляет собой объединение дисков и простых путей, аналогичен подслучаю простых кольцевых диаграмм из первого случая.

Рассмотренные случаи показывают, что $n\left|w_{0}\right|<\left|w_{0}\right|^{3}+4\left|w_{0}\right|^{3}$ или $n<5\left|w_{0}\right|^{2}$. Теорема доказана. 


\section{Список литературы}

1. Appel K., One Artin groups and Coxeter groups of large type. Contemp. Math. (1984) 33, 50-78.

2. Безверхний В. Н., Добрынина И. В., Решение проблемы сопряженности слов в группах Кокстера большого типа. Чебымевский сб. (2003) 4, №1(5), 10-33.

3. Линдон Р., Шупп П., Комбинаторная теория групn. Наука, Москва, 1980.

4. Безверхний В. Н., Добрынина И. В., Решение проблемы обобщенной сопряженности слов в группах Кокстера большого типа. Дискретная математика (2005) 17, №3, 123-145.

5. Безверхний В. Н., Добрынина И. В., Об элементах конечного порядка в группах Кокстера большого типа. Изв. Тульского гос. ун-та. Сер. Математика. Механика. Информатика (2003) 9, №1, $13-22$.

6. Безверхний В. Н., Добрынина И. В., Решение проблемы вхождения в циклическую подгруппу в группах Кокстера большого типа. Изв. Тульского гос. ун-та. Сер. Математика. Механика. Информатика (2004) 10, №1, 23-37.

7. Безверхний В. Н., Добрынина И. В., Решение проблемы слабой степенной сопряженности слов в группах Кокстера большого типа. Изв. Тульского гос. ун-та. Сер. Математика. Механика. Информатика (2004) 10, №1, 38-46. 